\title{
The Limits of Medical Trust in Mitigating COVID-19 Vaccine Hesitancy among Black Americans
}

J Gen Intern Med 36(11):3629-31

DOI: $10.1007 / \mathrm{s} 11606-021-06743-3$

(C) Society of General Internal Medicine 2021

\section{INTRODUCTION}

A highly anticipated COVID-19 vaccine has the potential to slow the pandemic in 2021. But a preponderance of misinformation, including conspiracy theories spreading through social media, has left much of the American public skeptical of vaccine candidates, and may undermine vaccine adherence. ${ }^{1}$ Up to $40 \%$ of Americans either do not intend to be vaccinated, or are unsure. ${ }^{2}$

Black Americans have borne a particularly disproportionate share of COVID-19 infections, ${ }^{3}$ and surveys have revealed higher rates of COVID-19 vaccine hesitancy among Black Americans relative to other racial/ethnic groups. ${ }^{2}$ Centuries of medical racism and subsequent medical mistrust among racial/ethnic minorities ${ }^{3}$ has left COVID-19 vaccine trials struggling to achieve diverse participation. This casts doubt on many communities' ability to eventually achieve herd immunity. ${ }^{1}$ Efforts to increase trust among Black Americans may help alleviate these problems, but the relationships between race, COVID-19 beliefs, trust, and vaccine hesitancy are complicated. ${ }^{2}$ A richer understanding of these dynamics is crucial for diversifying participation in clinical trials and reducing vaccine hesitancy. We used nationally representative survey data from June 2020 to test the hypothesis that Black race would interact with medical trust to undermine COVID19 vaccine willingness.

\section{METHODS}

The study was deemed exempt by the University of Miami institutional review board. Qualtrics, partnering with Lucid, administered a survey from June 4 to 17, 2020 (just before US COVID-19 cases spiked in July), to a nationally representative quota sample of $n=1040$ Americans that matched 2010 US Census records on sex, age, race, and income. The survey assessed participant demographics, political ideology, religiosity, and health and economic impacts of COVID-19, and contained psychometric scales used as unidimensional

Received October 21, 2020

Accepted March 17, 2021

Published online May 21, 2021 measures of perceived stress, conspiracy thinking (CT), denialism, and trust in health institutions (THI). Vaccine willingness was measured using a 5-point Likert scale, strongly disagree to strongly agree, to the question, "If a vaccine for COVID-19 becomes available I would be willing to take it." We did not ask about factors that might increase vaccine willingness. We used Stata 16 to fit a multivariable mixed effects Tobit regression model of COVID-19 vaccine willingness with errors clustered by US state. A 2 -sided $P$-value less than .05 was considered significant.

\section{RESULTS}

Only $63.5 \%$ of participants strongly agreed $(36.6 \%)$ or agreed $(26.8 \%)$ with being willing to take a COVID-19 vaccine, consistent with other surveys conducted in spring 2020, with significant variation by race/ethnicity: $70.4 \%$ for White, $61.5 \%$ for Hispanic/Latinx, and $44.3 \%$ for Black respondents (Fig. 1a). In the multivariable model, age-squared, household income, education, self-reported stress, and THI were all positively associated with vaccine willingness, while age, Black race, conservative political ideology, and CT were negatively associated with vaccine willingness (Table 1). Given the strong effect sizes for Black race, THI, and CT, we included interaction terms for Black and THI, and Black and CT. The Black-THI interaction term was negatively associated with vaccine willingness, meaning that the effect of THI on vaccine willingness was qualified by Black race. The marginal effects plot of vaccine willingness by THI (Fig. 1b) revealed that even at the highest levels of THI, Black respondents were significantly less willing to take a COVID-19 vaccine (i.e., more vaccine hesitant) than White respondents.

\section{DISCUSSION}

This study expands our knowledge of how race is related to COVID-19 vaccination hesitancy by adjusting for personal traits such as stress, conspiracy thinking, and medical trust. Higher medical trust was associated with lower vaccine hesitancy, but public health officials and the media risk conflating demographics, conspiracy thinking, and medical trust in understanding public COVID-19 vaccine perceptions. The COVID-19 vaccine hesitancy expressed by Black respondents 
a

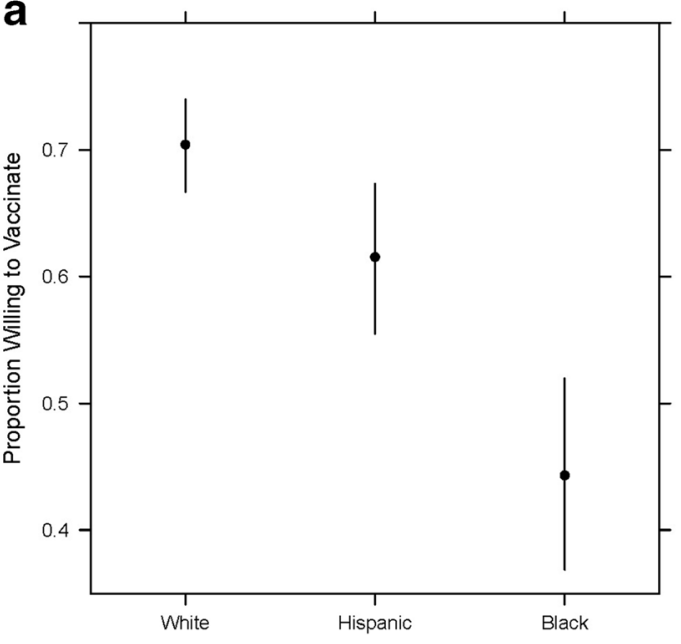

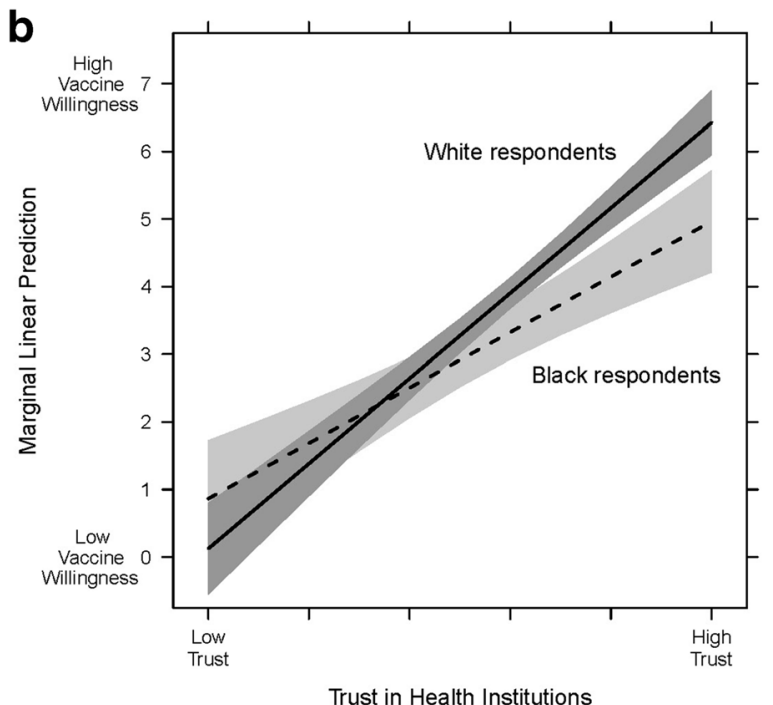

Fig. 1 a Proportion of White, Hispanic, and Black respondents who strongly agreed or agreed with the statement, "If a vaccine for COVID-19 becomes available I would be willing to take it" in June 2020, and b marginal linear prediction of vaccine willingness at different levels of trust in health institutions (THI) for Black and White respondents. In b, at the lowest levels of THI, everyone expressed low vaccine willingness, while at the highest levels of THI, Black respondents expressed significantly less willingness than White respondents.

is much greater than other racial immunization disparities across the life-course. ${ }^{4}$ The difference is likely attributable to structural racism, which requires the medical establishment to demonstrate its trustworthiness to Black Americans ${ }^{5}$ and recognize medical mistrust as a rightful adaptation to historical dehumanization in order to begin to mitigate vaccine hesitancy. ${ }^{3}$ Health education programs focused on building medical trust may underperform if they do not address structural racism. Because third-party survey respondent pools may be less generalizable to the Black and Hispanic population, ${ }^{6}$ the interaction between trust and vaccine willingness may be stronger than we detected. Difficult community dialogues may be a crucial first step toward engaging communities of color and promoting COVID-19 vaccine acceptance.

Table 1 Multivariable Mixed Effects Tobit Regression Model of COVID-19 Vaccine Willingness among 1032* Nationally Representative Survey Participants in June 2020

\begin{tabular}{|c|c|c|c|c|c|}
\hline Characteristic & $\boldsymbol{\beta}$ & SE & $Z$ & $P$-value & $95 \% \mathrm{CI}$ \\
\hline \multicolumn{6}{|l|}{ Individual-level fixed effects } \\
\hline Gender: male & .038 & .139 & .27 & .786 & -.235 to .311 \\
\hline Age & -.093 & .023 & -4.02 & $<.001$ & -.138 to -.047 \\
\hline Age -squared $^{-1}$ & .001 & .000 & 4.51 & $<.001$ & .001 to .002 \\
\hline Identify as: Black or African American & -.505 & .208 & -2.43 & .015 & -.913 to -.098 \\
\hline Identify as: Hispanic or Latino & -.071 & .163 & -.44 & .663 & -.391 to .248 \\
\hline Identify as: Asian American or Pacific Islander & -.360 & .350 & -1.03 & .304 & -1.046 to .326 \\
\hline Identify as: Native American or American Indian & -.482 & .434 & -1.11 & .267 & -1.333 to .369 \\
\hline Identify as: Other & -.477 & .551 & -.87 & .386 & -1.556 to .602 \\
\hline Annual household income & .090 & .046 & 1.97 & .049 & .000 to .180 \\
\hline Education & .151 & .052 & 2.89 & .004 & .048 to .253 \\
\hline Political ideology (liberal to conservative) & -.121 & .041 & -2.93 & .003 & -.201 to -.040 \\
\hline Religiosity & .035 & .055 & .63 & .530 & -.073 to .143 \\
\hline COVID-19 health impact score & .042 & .052 & .80 & .426 & -.061 to .144 \\
\hline COVID-19 economic disruption score & .286 & .149 & 1.92 & .054 & -.006 to .578 \\
\hline Perceived stress (PSS-4 score) & .073 & .024 & 3.03 & .002 & .026 to .120 \\
\hline Denialism scale & -.063 & .084 & -.75 & .450 & -.228 to .101 \\
\hline Conspiracy thinking scale & -.417 & .089 & -4.71 & $<.001$ & -.591 to -.244 \\
\hline Trust in health institutions scale & 1.171 & .089 & 13.14 & $<.001$ & .996 to 1.345 \\
\hline State with Republican Governor & -.054 & .134 & -.40 & .689 & -.316 to .209 \\
\hline Interaction term: Black $\times$ Conspiracy thinking & .095 & .197 & .48 & .630 & -.291 to .480 \\
\hline Interaction term: Black $\times$ Trust in health institutions & -.351 & .165 & -2.13 & .034 & -.675 to -.027 \\
\hline Constant & 4.875 & .620 & 7.86 & $<.001$ & 3.660 to 6.090 \\
\hline \multicolumn{6}{|l|}{ State-level effects } \\
\hline State-level constant & $3.10 \times 10^{-34}$ & $2.92 \times 10^{-19}$ & & & \\
\hline State-level error & 3.591 & .252 & & & 3.130 to 4.120 \\
\hline Model log likelihood $=-1466.0824$ & & & & & \\
\hline
\end{tabular}

*Note: 8 cases from the original 1040 sample had missing covariate data that led to case exclusion 
Justin Stoler, $\mathrm{PhD}, \mathrm{MPH}^{1,2}$

Adam M. Enders, $P h D^{3}$

Casey A. Klofstad, $P h D^{4}$

Joseph E. Uscinski, PhD ${ }^{4}$

${ }^{1}$ Department of Geography and Regional Studies, University of Miami,

Coral Gables, FL, USA

${ }^{2}$ Department of Public Health Sciences, Miller School of Medicine, University of Miami,

Miami, FL, USA

${ }^{3}$ Department of Political Science, University of Louisville,

Louisville, KY, USA

${ }^{4}$ Department of Political Science, University of Miami, Coral Gables, FL, USA

Corresponding Author: Justin Stoler, PhD, MPH; Department of Geography and Regional Studies, University of Miami, Coral Gables, FL, USA (e-mail: stoler@miami.edu).

Funding This work was supported by the University of Miami through a 2020 COVID-19 Rapid Response Award by the Office of the Vice-Provost for Research, and a 2020 COVID-19 Rapid Response Award by the College of Arts and Sciences, to Drs. Stoler, Klofstad, and Uscinski (PI). The sponsor had no role in the design and conduct of the study; collection, management, analysis, and interpretation of the data; preparation, review, or approval of the manuscript; and decision to submit the manuscript for publication.
Data Availability Study protocol, data set, and statistical code: Available from Dr. Justin Stoler (e-mail, stoler@miami.edu).

\section{Declarations:}

Conflict of Interest: The authors have disclosed no conflicts of interest.

\section{REFERENCES}

1. Schaffer DeRoo S, Pudalov NJ, Fu LY. Planning for a COVID-19 vaccination program. JAMA. 2020;323(24):2458-2459.

2. Fisher KA, Bloomstone SJ, Walder J, Crawford S, Fouayzi H, Mazor KM. Attitudes toward a potential SARS-CoV-2 vaccine: a survey of U.S. adults. Annals of Internal Medicine. 2020.

3. Manning KD. More than medical mistrust. The Lancet. 2020;396(10261):1481-1482.

4. Lu P-j, O'Halloran A, Williams WW, Lindley MC, Farrall S, Bridges CB Racial and ethnic disparities in vaccination coverage among adult populations in the U.S. American Journal of Preventive Medicine. 2015;49(6, Supplement 4):S412-S425.

5. Warren RC, Forrow L, Hodge DA, Truog RD. Trustworthiness before trust - Covid-19 vaccine trials and the Black community. $N$ Engl $\mathrm{J}$ Med. 2020;383(22):e121.

6. Kennedy C, Mercer A, Keeter S, Hatley N, McGeeney K, Gimenez A. Evaluating online nonprobability surveys. In. Washington, DC: Pew Research Center; 2016.

Publisher's Note: Springer Nature remains neutral with regard to jurisdictional claims in published maps and institutional affiliations. 